\title{
Effects of Lovastatin and Dietary Cholesterol on Sterol Homeostasis in Healthy Human Subjects
}

\author{
William C. Duane
}

Department of Medicine, Veterans Affairs Medical Center and University of Minnesota, Minneapolis, Minnesota 55417

\begin{abstract}
We measured biliary and fecal sterol outputs in 12 human subjects on a metabolic ward in four randomly allocated, 6-7 wk periods: (a) lovastatin (40 $\mathrm{mg}$ b.i.d.) + low cholesterol diet (mean $246 \mathrm{mg} / \mathrm{d}$ ), (b) lovastatin + high cholesterol diet (mean $1,071 \mathrm{mg} / \mathrm{d}),(c)$ low cholesterol diet alone, $(d)$ high cholesterol diet alone. In addition to lowering serum LDL cholesterol, lovastatin significantly lowered biliary secretion of cholesterol, fecal output of endogenous neutral sterols, cholesterol balance, and systemic cholesterol input (the sum of cholesterol synthesis and absorbed dietary cholesterol). The high cholesterol diet significantly lowered cholesterol balance, but significantly increased systemic cholesterol input and fecal output of acidic sterols. There was no significant interaction between lovastatin and dietary cholesterol for any parameter measured. Judging from these data, the primary action of lovastatin is to lower cholesterol synthesis and systemic cholesterol input, the main compensatory response being reduced biliary cholesterol secretion. Conversely, increased dietary cholesterol appears to increase systemic cholesterol input, the major compensatory response being increased bile acid synthesis. There appears to be no interaction between these two perturbations of systemic cholesterol input. (J. Clin. Invest. 1993. 92:911-918.) Key words: cholesterol $\bullet$ bile acids and salts $\bullet$ hydroxymethylglutaryl CoA reductases $\bullet$ atherosclerosis $\bullet$ cholelithiasis
\end{abstract}

\section{Introduction}

Lovastatin, an inhibitor of hydroxymethylglutaryl coenzymeA (HMG-CoA $)^{1}$ reductase, is a relatively new and very effective medication for lowering serum LDL cholesterol levels (1). It also reduces the cholesterol content of bile relative to bile acid and lecithin, a change that might theoretically be conducive to prevention or dissolution of cholesterol gallstones (2, 3 ). Two related drugs, simvastatin and pravastatin, appear to have similar effects (4-7).

The changes in sterol homeostasis accompanying or responsible for these beneficial effects remain ill-defined. Ironically,

Address correspondence and requests for reprints to Dr. William C. Duane, GI Section (111D), Veterans Affairs Medical Center, 1 Veterans Drive, Minneapolis, MN 55417.

Received for publication 20 November 1992 and in revised form 17 February 1993.

1. Abbreviations used in this paper: $\mathrm{HL}$, high dietary cholesterol and intake of lovastatin, $\mathbf{4 0 ~ m g ~ b . i . d . ; ~ H M G - C o A , ~ h y d r o x y m e t h y l g l u t a r y l ~}$ coenzyme-A. HN, high dietary cholesterol without lovastatin; LL, low dietary cholesterol and intake of lovastatin, $40 \mathrm{mg}$ b.i.d.; $\mathrm{LN}$, low dietary cholesterol without lovastatin.

The Journal of Clinical Investigation, Inc.

Volume 92, August 1993, 911-918 although these drugs were originally developed specifically because of their ability to inhibit cholesterol synthesis, it is not even clear that they produce sustained reductions in wholebody cholesterol synthesis in humans. Goldberg et al., using isotope dilution methods, reported that lovastatin did not alter cholesterol synthesis in a group of nine hypercholesterolemic subjects (8). In contrast, Vanhanen et al., using sterol balance techniques, found a reduction in mean cholesterol synthesis in subjects with heterozygous familial hypercholesterolemia taking pravastatin (9). Finally, an earlier study yielded an intermediate result: reduced cholesterol balance in three hypercholesterolemic subjects taking lovastatin, but no change in two others (10).

Other alterations in sterol homeostasis may also accompany lovastatin administration to human subjects. Our laboratory has documented a reduction in biliary cholesterol secretion on lovastatin (3), a finding that has been confirmed by another group for simvastatin (5). We have also reported reduced bile acid synthesis on lovastatin (11), and a similar effect has been reported for pravastatin (9). However, we have not been able to demonstrate a reduction with all methods for measuring bile acid synthesis, and other workers have reported little or no change in bile acid synthesis during treatment with lovastatin and related drugs $(10,12)$. Finally, one study has suggested that pravastatin might actually reduce cholesterol absorption in humans (13). However, in another study from the same laboratory, that effect was not demonstrated at a statistically significant level (9).

Besides biosynthesis, the only other input of cholesterol into body pools is from the diet. It is well known that dietary cholesterol exerts feedback control on cholesterol biosynthesis (14-18). The extent to which this homeostatic regulation is preserved during lovastatin treatment is unknown. Also unknown is the extent to which increased dietary cholesterol can substitute for any lovastatin-induced reduction in cholesterol biosynthesis with respect to maintaining either cholesterol secretion into bile or synthesis of bile acid.

In this study we sought to better define changes in sterol homeostasis induced by lovastatin and to answer some of these questions with regard to human subjects. The study was designed to rigorously control both dietary cholesterol (high or low) and lovastatin (on and off) as separate variables in a randomized block design. 12 subjects were each studied in four separate periods representing all possible combinations of these two variables.

\section{Methods}

12 male volunteers, ranging in age from 40 to $75 \mathrm{yr}$, were studied. All were without significant medical problems, as judged by previously published criteria (19), and all were shown to be free of gallstones by ultrasonography. After a detailed explanation of study procedures, each gave his written consent to participate. All study protocols were approved by committees overseeing use of human subjects in research 
at both the Minneapolis Veterans Affairs Medical Center and the University of Minnesota.

Except for one person (subject 8), who lived near the hospital, all subjects lived on the metabolic ward during the entire study. All subjects, including subject 8 , ate only meals served by the metabolic kitchen. Meals consisted of regular food selected and weighed to provide constant daily amounts of calories, cholesterol, fat (30\%), carbohydrate $(54 \%)$, and protein ( $16 \%)$. The menu was repeated on a weekly basis to provide overall consistency. Low cholesterol dietary intake was achieved by elimination of foods with high cholesterol content. High cholesterol intake was achieved by isocaloric substitution of five eggs per day in the low cholesterol menu.

All subjects were studied in four different 6-7 w periods separated by 3-7 d. For each subject the chronological order of periods was random. The four periods were designated as: (LN) low dietary cholesterol without lovastatin; (HN) high dietary cholesterol without lovastatin; (LL) low dietary cholesterol and intake of lovastatin, $40 \mathrm{mg}$ b.i.d.; (HL) high dietary cholesterol and intake of lovastatin, $40 \mathrm{mg}$ b.i.d.

In periods designated as low dietary cholesterol, mean $( \pm \mathrm{SD})$ cholesterol intake was $246 \pm 74 \mathrm{mg}$ per d. In periods designated as high dietary cholesterol, mean daily cholesterol intake was $1,071 \pm 100 \mathrm{mg}$ per $d$. Most of the variation was between, rather than within, subjects. Thus, the mean ( \pm SD) intrasubject difference between dietary cholesterol intake for the two low dietary cholesterol periods and for the two high dietary cholesterol periods was $1.5 \pm 10.9 \%$.

Subjects were weighed daily to assure that caloric intake was appropriate to maintain a steady state. Serum lipids were monitored weekly, using methods previously described. (3). For all subjects except 2 and 7, LDL cholesterol levels were calculated from the serum triglyceride level by standard methods, assuming a ratio of triglyceride/cholesterol in VLDL of 5.0. Because subjects 2 and 7 had elevated serum triglycerides, their serum VLDL was separated by ultracentrifugation, and the cholesterol/triglyceride ratio was determined using enzymatic methods $(20,21)$.

For the last $20 \mathrm{~d}$ of each period, subjects ingested $200 \mathrm{mg}$ chromic oxide three times daily as a nonabsorbable marker. For the last $10 \mathrm{~d}$ of each period, stool was quantitatively collected. Collections for each of the five 2-d intervals were homogenized with an equal volume of water. Aliquots of these homogenates were analyzed for neutral and acidic sterols as previously reported (22), using a modification of the method originally described by Miettinen, Ahrens, and Grundy (23). Total acidic sterol output $\left(A_{\mathrm{T}}\right)$ was calculated by multiplying daily chromium intake times the mean concentration ratio of acidic sterol/chromium in stool. Total neutral stool output $\left(N_{\mathrm{T}}\right)$ was calculated analogously from the mean concentration ratio of neutral sterol/chromium in stool.

On the last day of each period, measurements of biliary lipid secretion were performed by marker perfusion as previously outlined (3) using the method originally described by Grundy and Metzger (24).

Fractional cholesterol absorption $(F)$, endogenous neutral sterol output $\left(N_{\mathrm{E}}\right)$, cholesterol balance $(B)$, and systemic cholesterol input $(I)$ were calculated from daily dietary cholesterol intake $(D)$, hourly cholesterol secretion $(S), A_{\mathrm{T}}$, and $N_{\mathrm{T}}$ by the following formulas:

$F=\left(24 S+D-N_{\mathrm{T}}\right) /(24 S+D)$

$N_{\mathrm{E}}=N_{\mathrm{T}}-[(1-F)(D)]$

$B=D-A_{\mathrm{T}}-N_{\mathrm{T}}$

$I=(F)(D)-B=N_{\mathrm{E}}+A_{\mathrm{T}}$.

Statistical testing was performed using SAS software (SAS Institute, Cary, NC) on a personal computer (Northgate Computer Systems, Inc., Plymouth, MN) equipped with a 486DX microprocessor. All testing was by ANOVA with randomized block design followed by linear contrast analysis (25). This procedure permits comprehensive comparison of all periods with a common treatment (e.g., lovastatin) to all periods without the treatment to determine, for example, lovastatin effect. It also permits the more limited comparison of one period to another, which we refer to subsequently as "period pair" comparisons. Finally, it enables testing the null hypothesis for interaction between two treatments, in this case lovastatin and dietary cholesterol. Differences were designated as statistically significant when the two-sided $P$ value was less than 0.05 .

\section{Results}

Serum lipids. Serum lipid levels at the end of each treatment period for each subject are presented in Tables I and II. A summary of means and ANOVA testing for all measured parameters is presented in Table VII. Lovastatin lowered mean total cholesterol, LDL cholesterol, and triglyceride whether subjects were on the low or high cholesterol diet. Lovastatin also slightly increased mean HDL cholesterol. This change was statistically significant by ANOVA of the data set as a whole ( Table VII), but was significant by comparison of period pairs only when the diet was low in cholesterol (Table II). With that exception, the above changes were statistically significant whether judged by contrast analysis of period pairs (Tables I and II) or by contrast analysis of the entire data set for a lovastatin effect (Table VII).

Dietary cholesterol increased mean serum cholesterol and LDL cholesterol, but these changes were statistically significant only for LDL cholesterol and then only by contrast analysis of the data set as a whole (Table VII). Neither HDL cholesterol nor triglyceride levels were appreciably altered by increasing dietary cholesterol. Also, for none of the serum lipids was there a significant interaction between dietary cholesterol and lovastatin (Table VII).

Biliary lipid secretion. Table III presents all individual measurements of cholesterol and bile acid secretion into bile. Mean secretion rates and results of overall ANOVA testing for these two lipids as well as lecithin are presented in Table VII. Lovastatin lowered mean cholesterol secretion by $30 \%$ whether dietary cholesterol intake was low or high. These changes were statistically significant as judged, both by contrast analysis of period pairs or analysis of the data set as a whole ( Table VII). Diet had no effect on cholesterol secretion and there was nothing ap-

Table I. Serum Total and LDL Cholesterol Levels

\begin{tabular}{|c|c|c|c|c|c|c|c|c|}
\hline \multirow[b]{2}{*}{ Subject } & \multicolumn{4}{|c|}{ Total cholesterol } & \multicolumn{4}{|c|}{ LDL cholesterol } \\
\hline & $\mathrm{LN}$ & $\mathrm{HN}$ & LL & HL & LN & HN & LL & HL \\
\hline & \multicolumn{4}{|c|}{$m g / d l$} & \multicolumn{4}{|c|}{$m g / d l$} \\
\hline 1 & 216 & 217 & 151 & 152 & 145 & 146 & 87 & 87 \\
\hline 2 & 193 & 186 & 123 & 122 & 40 & 81 & 29 & 47 \\
\hline 3 & 192 & 181 & 119 & 121 & 132 & 123 & 64 & 65 \\
\hline 4 & 189 & 204 & 117 & 135 & 131 & 150 & 67 & 85 \\
\hline 5 & 205 & 215 & 138 & 144 & 133 & 131 & 47 & 59 \\
\hline 6 & 197 & 201 & 145 & 140 & 143 & 145 & 88 & 85 \\
\hline 7 & 232 & 278 & 201 & 205 & 132 & 163 & 112 & 120 \\
\hline 8 & 142 & 161 & 97 & 97 & 86 & 105 & 42 & 46 \\
\hline 9 & 313 & 318 & 196 & 191 & 245 & 251 & 133 & 128 \\
\hline 10 & 199 & 213 & 120 & 132 & 136 & 152 & 63 & 73 \\
\hline 11 & 268 & 301 & 150 & 165 & 195 & 227 & 81 & 101 \\
\hline 12 & 138 & 155 & 100 & 102 & 80 & 102 & 43 & 47 \\
\hline Mean & 207 & 219 & $138^{* \neq}$ & $142^{* \pm}$ & 133 & 148 & $71^{*}$ & $79^{* \neq}$ \\
\hline
\end{tabular}

* Significantly different from LN. ${ }^{\ddagger}$ Significantly different from HN. 
Table II. Serum HDL Cholesterol and Triglyceride Levels

\begin{tabular}{|c|c|c|c|c|c|c|c|c|}
\hline \multirow[b]{2}{*}{ Subject } & \multicolumn{4}{|c|}{ HDL cholesterol } & \multicolumn{4}{|c|}{ Triglyceride } \\
\hline & LN & $\mathrm{HN}$ & LL & HL & $\mathrm{LN}$ & HN & LL & HL \\
\hline & \multicolumn{4}{|c|}{$m g / d l$} & \multicolumn{4}{|c|}{$m g / d l$} \\
\hline 1 & 37 & 42 & 36 & 40 & 168 & 146 & 133 & 118 \\
\hline 2 & 26 & 32 & 33 & 33 & 631 & 360 & 303 & 206 \\
\hline 3 & 43 & 43 & 43 & 44 & 83 & 74 & 59 & 60 \\
\hline 4 & 37 & 34 & 37 & 34 & 104 & 96 & 63 & 78 \\
\hline 5 & 47 & 52 & 65 & 64 & 121 & 152 & 128 & 102 \\
\hline 6 & 35 & 37 & 42 & 41 & 91 & 91 & 71 & 66 \\
\hline 7 & 26 & 30 & 30 & 35 & 370 & 425 & 292 & 247 \\
\hline 8 & 37 & 34 & 38 & 33 & 87 & 107 & 83 & 93 \\
\hline 9 & 32 & 38 & 43 & 42 & 178 & 142 & 100 & 102 \\
\hline 10 & 33 & 36 & 35 & 36 & 147 & 123 & 105 & 111 \\
\hline 11 & 46 & 47 & 44 & 44 & 132 & 133 & 120 & 103 \\
\hline 12 & 44 & 37 & 43 & 44 & 69 & 74 & 70 & 50 \\
\hline Mean & 37 & 38 & $41^{*}$ & $41^{*}$ & 181 & 160 & $127^{*}$ & $111^{* \ddagger}$ \\
\hline
\end{tabular}

* Significantly different from LN. ${ }^{\ddagger}$ Significantly different from HN.

proaching a significant interaction between dietary cholesterol and lovastatin for cholesterol secretion.

Neither lovastatin nor dietary cholesterol intake had any appreciable effect on either bile acid or lecithin secretion rates. Moreover, there was no significant interaction between dietary cholesterol and lovastatin for either bile acid or lecithin secretion rate.

Fecal sterols and cholesterol absorption. Individual fecal acidic and total neutral sterol excretion rates are presented in Table IV. Lovastatin had no significant effect on acidic sterol output, whether by analysis of period pairs or the overall data set (Table VII). Increasing dietary cholesterol increased mean acidic sterol output both on and off lovastatin, although this change was significant by analysis of period pairs only when subjects were on lovastatin. There was also a highly significant overall effect of dietary cholesterol on acidic sterol by contrast analysis of the entire data set (Table VII). No significant interaction between dietary cholesterol and lovastatin (Table VII) was present.

Total neutral sterol output was consistently decreased by lovastatin and increased by the high cholesterol diet, whether examined by contrast analysis of period pairs (Table IV) or overall analysis of the entire data set (Table VII). There was no appreciable interaction between dietary cholesterol and lovastatin for neutral sterol output (Table VII).

Output of endogenous neutral sterols was consistently lowered by lovastatin, whether subjects were on the high or low cholesterol diet by both analysis of period pairs (Table V) or analysis of the entire data set (Table VII). Dietary cholesterol, however, had no appreciable effect on endogenous neutral sterol output by either mode of analysis. There was also no significant interaction between dietary cholesterol and lovastatin for endogenous neutral sterol output.

Fractional cholesterol absorption was not significantly affected by either dietary cholesterol or lovastatin, and there was no interaction of dietary cholesterol and lovastatin on cholesterol absorption (Tables V and VII).

Cholesterol balance was significantly reduced both by dietary cholesterol and by lovastatin, judging from both contrast analysis of period pairs and analysis of the entire data set ( Tables VI and VII). Moreover there was no suggestion of an interaction of dietary cholesterol and lovastatin on sterol balance (Table VII). Consistent with this lack of interaction, lovastatin significantly lowered sterol balance for periods of both high and low dietary cholesterol, and increasing dietary cholesterol significantly lowered cholesterol balance both on and off lovastatin (Table VI).

Systemic cholesterol input was significantly reduced by lovastatin in periods of both high and low dietary cholesterol ( Table VI). Contrast analysis of the entire data set indicated a significant lovastatin effect on systemic cholesterol input with no interaction between dietary cholesterol and lovastatin ( Ta-

Table III. Biliary Cholesterol and Bile Acid Secretion

\begin{tabular}{|c|c|c|c|c|c|c|c|c|}
\hline \multirow[b]{2}{*}{ Subject } & \multicolumn{4}{|c|}{ Cholesterol secretion } & \multicolumn{4}{|c|}{ Bile acid secretion } \\
\hline & $\mathrm{LN}$ & $\mathrm{HN}$ & LL & $\mathrm{HL}$ & LN & $\mathrm{HN}$ & LL & $\mathrm{HL}$ \\
\hline & \multicolumn{4}{|c|}{$\mu \mathrm{mol} / \mathrm{h}$} & \multicolumn{4}{|c|}{$\mu m o l / h$} \\
\hline 1 & 139 & 319 & 112 & 109 & 1,604 & 3,141 & 1,352 & 1,144 \\
\hline 2 & 103 & 106 & 107 & 96 & 833 & 812 & 1,141 & 690 \\
\hline 3 & 120 & 123 & 94 & 83 & 1,161 & 1,419 & 1,174 & 999 \\
\hline 4 & 104 & 94 & 89 & 55 & 1,495 & 1,215 & 1,911 & 1,285 \\
\hline 5 & 272 & 179 & 141 & 127 & 1,910 & 1,103 & 1,462 & 1,643 \\
\hline 6 & 326 & 193 & 155 & 230 & 1,767 & 2,063 & 1,141 & 1,540 \\
\hline 7 & 145 & 153 & 102 & 137 & 1,880 & 1,549 & 1,761 & 1,974 \\
\hline 8 & 134 & 112 & 106 & 110 & 1,854 & 1,601 & 2,009 & 1,181 \\
\hline 9 & 97 & 146 & 93 & 89 & 952 & 1,567 & 1,411 & 1,488 \\
\hline 10 & 93 & 108 & 54 & 77 & 1,154 & 824 & 761 & 977 \\
\hline 11 & 115 & 101 & 61 & 114 & 1,369 & 1,380 & 1,020 & 1,103 \\
\hline 12 & 114 & 197 & 104 & 74 & 786 & 1,937 & 1,685 & 870 \\
\hline Mean & 147 & 153 & $102^{* \ddagger}$ & $108^{* \ddagger}$ & 1,397 & 1,551 & 1,402 & 1,241 \\
\hline
\end{tabular}

* Significantly different from LN. ${ }^{\ddagger}$ Significantly different from HN. 
Table IV. Fecal Acidic and Total Neutral Sterols

\begin{tabular}{|c|c|c|c|c|c|c|c|c|}
\hline \multirow[b]{2}{*}{ Subject } & \multicolumn{4}{|c|}{ Acidic sterols } & \multicolumn{4}{|c|}{ Neutral sterols } \\
\hline & LN & HN & LL & HL & $\mathbf{L N}$ & HN & LL & HL \\
\hline & \multicolumn{4}{|c|}{$\mu \mathrm{mol} / \mathrm{d}$} & \multicolumn{4}{|c|}{$\mu \mathrm{mol} / \mathrm{d}$} \\
\hline 1 & 650 & 788 & 609 & 811 & 2,072 & 3,725 & 1,715 & 3,919 \\
\hline 2 & 1,320 & 941 & 1,178 & 1,436 & 2,262 & 3,916 & 1,568 & 3,546 \\
\hline 3 & 953 & 970 & 799 & 821 & 2,622 & 4,475 & 2,491 & 4,541 \\
\hline 4 & 1,114 & 1,673 & 950 & 1,672 & 2,142 & 4,427 & 2,255 & 3,258 \\
\hline 5 & 1,015 & 1,263 & 974 & 976 & 1,847 & 3,608 & 1,191 & 2,793 \\
\hline 6 & 1,610 & 2,014 & 2,033 & 2,368 & 4,227 & 5,207 & 2,675 & 3,503 \\
\hline 7 & 1,243 & 1,312 & 1,098 & 1,559 & 3,240 & 5,086 & 2,785 & 3,471 \\
\hline 8 & 1,794 & 1,475 & 1,382 & 1,776 & 1,845 & 4,634 & 1,914 & 4,057 \\
\hline 9 & 890 & 1,001 & 843 & 998 & 2,539 & 2,757 & 2,227 & 3,308 \\
\hline 10 & 1,062 & 1,362 & 1,078 & 1,238 & 1,406 & 2,426 & 1,310 & 2,392 \\
\hline 11 & 721 & 960 & 825 & 842 & 1,605 & 2,894 & 1,620 & 2,962 \\
\hline 12 & 2,282 & 2,178 & 1,703 & 2,221 & 2,798 & 3,400 & 1,843 & 3,603 \\
\hline Mean & 1,221 & 1,328 & 1,123 & $1,393^{8}$ & 2,384 & $3,880^{*}$ & $1,966^{* \ddagger}$ & $3,446^{* \neq 5}$ \\
\hline
\end{tabular}

* Significantly different from LN. ${ }^{\ddagger}$ Significantly different from HN; ${ }^{\S}$ significantly different from LL.

ble VII). Dietary cholesterol, on the other hand, significantly increased systemic cholesterol input regardless of whether subjects were taking lovastatin or not (Tables VI and VII).

Analyses excluding hyperlipidemic subjects. Because two of our subjects were hypertriglyceridemic (subjects 2 and 7) and two others were hypercholesterolemic (subjects 9 and 11), we performed complete contrast analysis separately eliminating both these pairs of subjects. In both cases there was no change in statistically significant results for any parameter compared to analysis of the data with all subjects included (Table VII).

\section{Discussion}

Study design. Quantitative characterization of sterol homeostasis and its determinants in human subjects remains a formida- ble challenge, in part because of variability. We attempted to manage and minimize variability, first by randomizing treatment periods and second by standardizing study conditions. All subjects ate only food served by the metabolic kitchen and, with one exception, all lived on the metabolic ward throughout the study. In addition, to minimize random measurement variability, fecal sterol measurements were made on quantitative collections over a 10-d period, and measurements of biliary secretion were made on eight 1 -h collections. To reduce biological variability, we attempted to recruit healthy subjects without gallstones and with fairly normal serum lipid levels. Because of the difficulty in recruiting subjects for such demanding studies, the latter efforts were only partially successful. Thus, two of our subjects had hypertriglyceridemia and two others had hypercholesterolemia. However, statistical analysis of the data omit-

Table V. Cholesterol Absorption and Endogenous Neutral Sterols

\begin{tabular}{|c|c|c|c|c|c|c|c|c|}
\hline \multirow[b]{2}{*}{ Subject } & \multicolumn{4}{|c|}{ Cholesterol absorption } & \multicolumn{4}{|c|}{ Endogenous neutral sterols } \\
\hline & $\mathbf{L N}$ & $\mathbf{H N}$ & $\mathbf{L L}$ & HL & $\mathbf{L N}$ & $\mathrm{HN}$ & $\mathbf{L L}$ & HL \\
\hline & \multicolumn{4}{|c|}{ fraction } & \multicolumn{4}{|c|}{$\mu \mathrm{mol} / \mathrm{d}$} \\
\hline 1 & 0.45 & 0.63 & 0.45 & 0.23 & 1,844 & 2,815 & 1,487 & 2,018 \\
\hline 2 & 0.21 & 0.21 & 0.47 & 0.26 & 1,965 & 2,027 & 1,382 & 1,719 \\
\hline 3 & 0.24 & 0.21 & 0.11 & 0.04 & 2,212 & 2,342 & 2,011 & 1,912 \\
\hline 4 & 0.29 & 0.11 & 0.19 & 0.20 & 1,781 & 2,017 & 1,743 & 1,062 \\
\hline 5 & 0.73 & 0.48 & 0.70 & 0.51 & 1,734 & 2,245 & 1,017 & 1,502 \\
\hline 6 & 0.50 & 0.28 & 0.36 & 0.57 & 3,922 & 3,364 & 2,385 & 2,406 \\
\hline 7 & 0.22 & 0.21 & 0.13 & 0.43 & 2,718 & 2,886 & 2,154 & 1,880 \\
\hline 8 & 0.55 & 0.24 & 0.42 & 0.31 & 1,456 & 2,058 & 1,481 & 1,833 \\
\hline 9 & 0.15 & 0.55 & 0.24 & 0.34 & 1,982 & 1,582 & 1,715 & 1,415 \\
\hline 10 & 0.52 & 0.55 & 0.35 & 0.49 & 1,078 & $1,17.0$ & 848 & 959 \\
\hline 11 & 0.55 & 0.46 & 0.30 & 0.48 & 1,255 & 1,310 & 1,027 & 1,436 \\
\hline 12 & 0.26 & 0.57 & 0.48 & 0.27 & 2,048 & 2,048 & 1,307 & 1,315 \\
\hline Mean & 0.39 & 0.37 & 0.35 & 0.34 & 2,000 & 2,155 & $1,546^{* \neq}$ & $1,622^{* \neq}$ \\
\hline
\end{tabular}

* Significantly different from $\mathrm{LN}$. ${ }^{\ddagger}$ Significantly different from HN. 
Table VI. Fecal Sterol Balance and Systemic Cholesterol Input

\begin{tabular}{|c|c|c|c|c|c|c|c|c|}
\hline \multirow[b]{2}{*}{ Subject } & \multicolumn{4}{|c|}{ Sterol balance } & \multicolumn{4}{|c|}{ Systemic cholesterol input } \\
\hline & LN & $\mathrm{HN}$ & LL & HL & $\mathbf{L N}$ & HN & $\mathbf{L L}$ & HL \\
\hline & \multicolumn{4}{|c|}{$\mu \mathrm{mol} / \mathrm{d}$} & \multicolumn{4}{|c|}{$\mu \mathrm{mol} / \mathrm{d}$} \\
\hline 1 & -2309 & -2035 & -1912 & -2252 & 2,494 & 3,603 & 2,097 & 2,829 \\
\hline 2 & -3209 & -2470 & -2398 & -2517 & 3,286 & 2,968 & 2,561 & 3,156 \\
\hline 3 & -3039 & -2747 & -2749 & -2618 & 3,165 & 3,312 & 2,811 & 2,734 \\
\hline 4 & -2746 & -3382 & -2570 & -2178 & 2,895 & 3,690 & 2,693 & 2,735 \\
\hline 5 & -2435 & -2251 & -1584 & -1149 & 2,750 & 3,509 & 1,992 & 2,479 \\
\hline 6 & -5228 & -4678 & -4254 & -3346 & 5,532 & 5,378 & 4,419 & 4,775 \\
\hline 7 & -3812 & -3597 & -3160 & -2229 & 3,962 & 4,199 & 3,252 & 3,440 \\
\hline 8 & -2774 & -2738 & -2548 & -2609 & 3,251 & 3,533 & 2,863 & 3,610 \\
\hline 9 & -2771 & -1150 & -2399 & -1428 & 2,873 & 2,583 & 2,559 & 2,414 \\
\hline 10 & -1784 & -1002 & -1679 & -844 & 2,140 & 2,532 & 1,927 & 2,197 \\
\hline 11 & -1552 & -906 & -1592 & -892 & 1,977 & 2,271 & 1,852 & 2,278 \\
\hline 12 & -4073 & -2445 & -2521 & -2693 & 4,330 & 4,226 & 3,011 & 3,536 \\
\hline Mean & -2978 & $-2450^{*}$ & $-2447^{*}$ & $-2063^{* \neq \S}$ & 3,221 & $3,484^{*}$ & $2,670^{* \neq}$ & $3,015^{\text {} \$}$ \\
\hline
\end{tabular}

* Significantly different from LN. ${ }^{\ddagger}$ Significantly different from HN; ${ }^{\S}$ significantly different from LL.

ting these two pairs of subjects yielded results essentially identical to those with all subjects included.

Serum lipids. With respect to separate effects of lovastatin and dietary cholesterol on serum lipids, the results confirm the findings of many previous studies $(1,8,15-18)$. Keys has suggested that serum total cholesterol rises as a function of the square root of dietary cholesterol, so that a sharper incremental rise occurs below dietary cholesterol levels of $\sim 300 \mathrm{mg} / \mathrm{d}$ than above that level (26). Hegsted disputes this, suggesting instead that the relationship is linear (27). To the extent that the Keys model is the better fit, we might have seen a disproportionately greater effect of dietary cholesterol by providing even less cholesterol in the low cholesterol diet.

There has been very little previous study of the potential interaction between lovastatin and dietary cholesterol with respect to serum lipid levels, although one report did show no interaction between dietary fat and lovastatin (28). The present study shows that there is no interaction between dietary cholesterol and lovastatin. In our subjects the effects of lovastatin on all measured serum lipids were independent of dietary

Table VII. Summary of Means and ANOVA Testing for Overall Effects

\begin{tabular}{|c|c|c|c|c|c|c|c|}
\hline \multirow[b]{2}{*}{ Variable } & \multicolumn{4}{|c|}{ Means } & \multicolumn{3}{|c|}{ Overall effects } \\
\hline & $\mathbf{L N}$ & HN & LL & HL & Diet $^{*}$ & Lov $^{*}$ & Int \\
\hline & & & & & & Pvalue & \\
\hline \multicolumn{8}{|l|}{ Serum } \\
\hline Cholesterol & 207 & 219 & 381 & 142 & 0.099 & 0.0001 & 0.404 \\
\hline LDL cholesterol & 133 & 148 & 71 & 79 & 0.047 & 0.0001 & 0.484 \\
\hline HDL cholesterol & 37 & 38 & 41 & 41 & 0.406 & 0.0038 & 0.454 \\
\hline Triglycerides & 181 & 160 & 127 & 111 & 0.231 & 0.0019 & 0.857 \\
\hline \multicolumn{8}{|l|}{ Biliary secretion } \\
\hline Cholesterol & 147 & 153 & 102 & 108 & 0.588 & 0.0005 & 0.960 \\
\hline Lecithin & 415 & 493 & 392 & 416 & 0.316 & 0.323 & 0.589 \\
\hline Bile salt & 1,397 & 1,551 & 1,402 & 1,241 & 0.974 & 0.189 & 0.174 \\
\hline \multicolumn{8}{|l|}{ Fecal sterols } \\
\hline Acidic & 1,221 & 1,328 & 1,123 & 1,393 & 0.0007 & 0.743 & 0.115 \\
\hline Total neutral & 2,384 & 3,880 & 1,966 & 3,446 & 0.0001 & 0.0037 & 0.954 \\
\hline Endogenous neutral & 2,000 & 2,155 & 1,546 & 1,622 & 0.194 & 0.0001 & 0.647 \\
\hline Cholesterol absorption & 0.39 & 0.37 & 0.35 & 0.34 & 0.795 & 0.365 & 0.914 \\
\hline (-Cholesterol balance) & 2,978 & 2,450 & 2,447 & 2,063 & 0.0005 & 0.0004 & 0.546 \\
\hline Systemic cholesterol input & 3,221 & 3,484 & 2,670 & 3,015 & 0.008 & 0.0001 & 0.617 \\
\hline
\end{tabular}

Serum lipids are in $\mathrm{mg} / \mathrm{dl}$, biliary lipids in $\mu \mathrm{mol} / \mathrm{h}$, fecal sterols, cholesterol balance, and cholesterol input in $\mu \mathrm{mol} / \mathrm{d}$, and cholesterol absorption in percent.

* Diet, dietary cholesterol. ${ }^{\ddagger}$ Lov, lovastatin. ${ }^{8}$ Int, interaction between dietary cholesterol and lovastatin. 
cholesterol intake, and any effect of dietary cholesterol was independent of lovastatin administration. This implies that the compensatory mechanisms, which tend to minimize elevation of serum cholesterol on a high cholesterol diet, remain fully intact despite the inhibitory effect of lovastatin on HMG-CoA reductase.

Biliary cholesterol secretion. We have previously reported an $\sim 30 \%$ reduction in biliary cholesterol secretion on lovastatin (3), a finding confirmed by others (5). The present study also strongly confirms that observation, showing a highly significant lovastatin effect with respect to biliary cholesterol secretion (Table VII). This finding was independently supported by a similar reduction in fecal output of endogenous neutral sterols, which, in the absence of altered cholesterol absorption, should be equivalent to output of cholesterol into bile plus cholesterol sloughed in intestinal epithelial cells. Moreover, both direct biliary measurements and the less direct fecal measurements showed similar reductions of biliary cholesterol secretion induced by lovastatin in periods of low vs. high dietary cholesterol intake (Table VII).

Dietary cholesterol itself had no significant effect on biliary cholesterol secretion (Tables III and VII). We can find only three studies in which the effects of dietary cholesterol on biliary cholesterol secretion were assessed by direct measurements on bile. The first two of these studies reported stimulation of cholesterol secretion by dietary cholesterol $(14,29)$, but both were much smaller than the current study (one and three subjects, respectively), and the larger of the two studies evaluated subjects in a somewhat unphysiological situation after cholecystectomy and placement of a T-tube (29). The third, which was a study by Everson et al. of 13 outpatient subjects on and off premarin (30), reported an increase in cholesterol secretion by patients on high cholesterol diets, but the overall change was of borderline statistical significance $(P<0.07)$, and the predominant change occurred on premarin. In the present study, not only did direct measurements of biliary cholesterol output demonstrate no effect of dietary cholesterol (Table VII), but this observation was confirmed independently by measurements of fecal endogenous neutral sterols (Tables V and VII). Moreover, this finding is in essential agreement with those of Everson et al. in subjects off premarin (30). We conclude that, with the exception of concurrent premarin treatment, dietary cholesterol has little or no appreciable influence on biliary cholesterol secretion.

Cholesterol absorption. Surprisingly, studies in rabbits have suggested that pravastatin and simvastatin might lower cholesterol absorption $(31,32)$. Moreover, in a study available only in abbreviated form, Miettinen and co-workers reported significant lowering of cholesterol absorption efficiency in hypercholesterolemic subjects taking pravastatin for more than 6 mo (13). Our results show no significant change in cholesterol absorption efficiency after $6 \mathrm{wk}$ of lovastatin. Of course, it is possible that the effects of lovastatin on cholesterol absorption might have become more pronounced had lovastatin been continued for $6 \mathrm{mo}$, as in the preliminary study of Miettinen et al. (13). On the other hand, effects of HMG-CoA reductase inhibitors on serum lipids are at or near a plateau by $6 \mathrm{wk}$ of treatment and show little tendency to change in the ensuing $10 \mathrm{mo}$ $(1,11,33)$. It should also be noted that Miettinen measured cholesterol absorption by the fecal isotope ratio method after simultaneous administration of $\left[{ }^{14} \mathrm{C}\right]$ cholesterol and $\left[{ }^{3} \mathrm{H}\right]-$ sitosterol, whereas we determined absorption from direct mass measurements on bile and feces. To the extent that there is isotope exchange unaccompanied by mass transfer, there may be differences in absorption values returned by these two methods (34).

We also found no effect of dietary cholesterol on absorption efficiency of cholesterol. Some previous studies are in agreement with this finding $(15,16,18)$, while others have reported a slight decrease in absorption efficiency with increasing dietary cholesterol $(17,30,35)$. It has been suggested that part of the reason for this discrepancy is that absorption efficiency is affected predominantly when dietary cholesterol greatly exceeds $1 \mathrm{~g} / \mathrm{d}$ (14), which was not the case in the present study. It has further been suggested that use of the dual isotope technique, which has been used to measure absorption in most studies, may be misleading when dietary cholesterol is increased because of isotope exchange $(34,35)$. This problem was avoided in this study by determining absorption through direct mass measurements. Thus, our data lend support to those who argue that dietary cholesterol of up to $\sim 1 \mathrm{~g} / \mathrm{d}$ does not appreciably affect absorption efficiency.

Fecal acidic sterols. We found that lovastatin had no appreciable effect on fecal acidic sterol output, which in the steadystate is a measure of bile acid synthesis (Tables IV and VII). In a previous study, using isotope dilution methodology, we also found no significant change in bile acid synthesis during lovastatin treatment (3). Moreover, activity of hepatic $7 \alpha$-hydroxylase in human liver is apparently not significantly altered by pravastatin treatment (12). On the other hand, Miettinen et al. reported a significant reduction in fecal sterol output in hypercholesterolemic subjects treated with pravastatin (9). In addition, our own laboratory, measuring release of ${ }^{14} \mathrm{CO}_{2}$ from $\left[{ }^{14} \mathrm{C}-26\right]$ cholesterol, found a significantly lower rate of bile acid synthesis in subjects taking lovastatin for $1 \mathrm{mo}(11)$. This latter study may be particularly instructive in that bile acid synthesis by ${ }^{14} \mathrm{CO}_{2}$ output was reduced to a greater extent and much more consistently after a single dose of lovastatin than after 1 mo of regular lovastatin. Thus, reduced bile acid synthesis may be an early adaptation to reduced cholesterol synthesis, but one that is later partially or completely supplanted by reduced cholesterol secretion into bile. It remains possible that long-term usage of lovastatin reduces bile acid synthesis, but if so, the reduction is apparently small enough to be detected only inconsistently.

Our data also reveal a highly significant effect of dietary cholesterol on output of acidic sterols ( Table VII). Several studies have reported increased synthesis of bile acids after increased cholesterol intake $(16,35)$ while others have reported no effect $(14,15,30)$. The present study contains more comparisons of high and low cholesterol diets in subjects studied on a metabolic ward than any single previous report. It also seems fair to say that our study was carried out and analyzed as rigorously as any previous study. As a result our observations lend strong support to those who contend that increasing dietary cholesterol to the level of about a gram per day increases bile acid synthesis.

Sterol balance. In the present study, lovastatin induced a highly significant reduction in sterol balance, the steady-state equivalent of cholesterol synthesis (Table VII). This finding is in agreement with the results of Vanhanen et al. (9). Grundy and Bilheimer also found reduced sterol balance in three subjects taking lovastatin, although not in two others (10). However, Goldberg et al., using isotope dilution techniques, re- 
ported no significant reduction in cholesterol production in nine hypercholesterolemic subjects (8). It is possible that hypercholesterolemic subjects respond differently to lovastatin than do normals. Indeed our two subjects with elevated serum cholesterols ( subjects 9 and 11 ) seemed to have less consistent changes in sterol balance on lovastatin than the group as a whole. On the other hand, the positive study of Vanhanen et al. noted above, was performed entirely on hypercholesterolemic patients (9). In addition, because Goldberg et al. treated their subjects with lovastatin for $15 \mathrm{mo}$, it is possible that cholesterol synthesis is reduced after 6-7 wk of treatment, but not after more prolonged treatment. As noted above, however, that possibility seems unlikely, based on the rapid plateau of serum lipids on lovastatin with little tendency for those changes to reverse after nearly $1 \mathrm{yr}$ of treatment (1). Finally, it should be noted that in the study of Goldberg et al. measurements on lovastatin were compared to measurements in the same subjects off lovastatin. However, the interval between studies was 3-8 yr, necessitating correction for the known effect of aging and weight change on cholesterol synthesis (36). Because many things can change in $8 \mathrm{yr}$, it is likely that this study design was not as sensitive in detecting differences as one in which subjects were studied only a few weeks apart. In any case, our data strongly indicate that lovastatin reduces cholesterol synthesis in normocholesterolemic subjects and that this reduction is maintained for at least $6 \mathrm{wk}$. It remains possible that hypercholesterolemic subjects do not respond in this way to lovastatin, although that would leave unexplained their persistent reduction in serum cholesterol levels.

Increasing dietary cholesterol also resulted in a highly significant reduction in sterol balance (Table VII). This finding is in general agreement with most previous studies (15-18), although there are apparently individuals who are relatively resistant to this feedback mechanism as judged from our own data (Table VI) and those of others $(14,37)$.

There was no interaction between dietary cholesterol and lovastatin with respect to sterol balance. Thus, feedback inhibition of cholesterol synthesis by dietary cholesterol remained fully intact despite demonstrable inhibition of cholesterol synthesis by lovastatin. Conversely, lovastatin remained able to further inhibit cholesterol synthesis even in the face of active feedback inhibition by dietary cholesterol.

Systemic cholesterol input. Finally, we calculated systemic cholesterol input, which represents the total amount of new cholesterol presented each day to the liver for disposal. There was a highly significant decrease in systemic cholesterol input induced by lovastatin, which was eliminated largely by a decrease in biliary cholesterol secretion (Table VII). Conversely, there was a highly significant increase in systemic cholesterol input induced by high levels of dietary cholesterol, which was eliminated largely by increased conversion to bile acid (Table VII). These changes in systemic cholesterol input presumably are seen by the liver as a need to either conserve cholesterol, in the case of lovastatin, or to eliminate cholesterol, in the case of high dietary cholesterol. Part of the liver's response is then to alter cholesterol flux into and out of the serum pool. Thus, decreased systemic input of cholesterol by lovastatin can be viewed as the initial stimulus leading to stimulation of LDL receptor activity (12) and decreased hepatic production of LDL (38), with subsequent reduction in serum cholesterol levels. Conversely, increased systemic input of cholesterol with cholesterol feeding leads to reduction in hepatic uptake (39,
40 ) and increased hepatic production of LDL (41) with eventual increase in serum LDL cholesterol.

Fortuitously, mean cholesterol synthesis rates were identical in periods $\mathrm{LL}$ and $\mathrm{HN}$, although systemic cholesterol input was much higher in HN because of dietary intake (Table VII). A similar increase in mean cholesterol input compared to period $\mathrm{LL}$ was present in period $\mathrm{LN}$, but in that case the increase was all from cholesterol biosynthesis (Table VII). Compared to period LL, either increasing only dietary cholesterol (period HN) or increasing only biosynthetic cholesterol (period LN) both markedly and similarly increased serum total cholesterol, LDL cholesterol, and biliary cholesterol secretion (Table VII). Thus, providing the other input was controlled, there was no apparent functional difference in dietary vs. biosynthetic input of cholesterol. In the absence of any such control, consumption of cholesterol has a much less pronounced effect on serum and biliary cholesterol because of a compensatory decrease in cholesterol synthesis, and, to a lesser extent, an increase in bile acid synthesis.

\section{Acknowledgments}

I wish to acknowledge the dedicated organizational and technical work of Ms. Cathy Pinther-Evans, Ann McHale, Margaret Jordan, and Linda Nelson, without which these studies would not have been possible.

Financial support was provided by the Department of Veterans Affairs and the National Institutes of Health (grant R01-DK42433).

\section{References}

1. Bradford, R. H., C. L. Shear, A. N. Chremos, F. A. Franklin, D. T. Nash, D. P. Hurley, C. A. Dujovne, J. L. Pool, H. Schnaper, M. Hesney, and A. Langendorfer. 1991. Expanded clinical evaluation of lovastatin (EXCEL) study results. III. Efficacy in modifying lipoproteins and implications for managing patients with moderate hypercholesterolemia. Am. J. Med. 91 (1B):18S-24S.

2. Freeman, M. L., W. F. Prigge, D. B. Hunninghake, W. C. Duane, and R. L. Gebhard. 1988. Intestinal HMG-CoA reductase activity is low in hypercholesterolemic patients and is further decreased with lovastatin therapy. J. Lipid Res. 29:839-845.

3. Mitchell, J. C., G. M. Logan, B. G. Stone, and W. C. Duane. 1991. Effects of lovastatin on biliary lipid secretion and bile acid metabolism in humans. J. Lipid Res. 32:71-78.

4. Duane, W. C., D. B. Hunninghake, M. L. Freeman, P. A. Pooler, L. A. Schlasner, and R. L. Gebhard. 1988. Simvastatin, a competitive inhibitor of HMG-CoA reductase, lowers cholesterol saturation index of gallbladder bile. Hepatology. 8:1147-1150.

5. Mazzella, G., P. Parini, D. Festi, F. Bazzoli, R. Aldini, A. Roda, D. Tonelli, A. Cipolla, A. Salzetta, and E. Roda. 1992. Effect of simvastatin, ursodeoxycholic acid and simvastatin plus ursodeoxycholic acid on biliary lipid secretion and cholic acid kinetics in nonfamilial hypercholesterolemia. Hepatology. 15:10721078.

6. Hoogerbrugge-vd Linden, N., F. W. M. de Rooy, H. Jansen, and M. vanBlankenstein. 1990. Effect of pravastatin on biliary lipid composition and bile acid synthesis in familial hypercholesterolaemia. Gut. 31:348-350.

7. Smit, J. W., K. J. Van Erpecum, M. F. J. Stolk, R. A. Geerdink, O. J. J. Cluysenaer, D. Willem Erkelens, and G. P. Van Berge Henegouwen. 1992. Successful dissolution of cholesterol gallstone during treatment with pravastatin. Gastroenterology. 103:1068-1070.

8. Goldberg, I. J., S. Holleran, R. Ramakrishnan, M. Adams, R. H. Palmer, R. B. Dell, and D. W. Goodman. 1990. Lack of effect of lovastatin therapy on the parameters of whole-body cholesterol metabolism. J. Clin. Invest. 86:801-808.

9. Vanhanen, H., Y. A. Kesäniemi, and T. A. Miettinen. 1992. Pravastatin lowers serum cholesterol, cholesterol-precursor sterols, fecal steroids, and cholesterol absorption in man. Metab. Clin. Exp. 41:588-595.

10. Grundy, S. M., and D. W. Bilheimer. 1984. Inhibition of 3-hydroxy-3methylglutaryl-CoA reductase by mevinolin in familial hypercholesterolemia heterozygotes: effects on cholesterol balance. Proc. Natl. Acad. Sci. USA. 81:25382542.

11. Mitchell, J. C., B. G. Stone, G. M. Logan, and W. C. Duane. 1991. Role of cholesterol synthesis regulation of bile acid synthesis and biliary cholesterol secretion in humans. J. Lipid Res. 32:1143-1149. 
12. Reihner, E., M. Rudling, D. Stahlberg, L. Berglund, S. Ewerth, I. Bjorkhem, K. Einarsson, and B. Angelin. 1990. Influence of pravastatin, a specific inhibitor of HMG-CoA reductase, on hepatic metabolism of cholesterol. $N$. Engl. J. Med. 323:224-228.

13. Miettinen, T. A. 1991. Inhibition of cholesterol absorption by HMG-CoA reductase inhibitor. Eur. J. Clin. Pharmacol. 40(Suppl. 1):S19-S21.

14. Quintao, E., S. M. Grundy, and E. H. Ahrens, Jr. 1971. Effects of dietary cholesterol on the regulation of total body cholesterol in man. J. Lipid Res. 12:233-247.

15. Nestel, P. J., and A. Poyser. 1976. Changes in cholesterol synthesis and excretion when cholesterol intake is increased. Metab. Clin. Exp. 25:1591-1599.

16. Lin, D. S., and W. E. Connor. 1980. The long term effects of dietary cholesterol upon the plasma lipids, lipoproteins, cholesterol absorption, and the sterol balance in man: the demonstration of feedback inhibition of cholesterol biosynthesis and increased bile acid excretion. J. Lipid Res. 21:1042-1052.

17. McNamara, D. J., R. Kolb, T. S. Parker, H. Batwin, P. Samuel, C. D. Brown, and E. H. Ahrens, Jr. 1987. Heterogeneity of cholesterol homeostasis in man. J. Clin. Invest. 79:1729-1739.

18. McMurry, M. P., W. E. Connor, D. S. Lin, M. T. Cerqueira, and S. L. Connor. 1985. The absorption of cholesterol and the sterol balance in the Tarahumara Indians of Mexico fed cholesterol-free and high cholesterol diets. Am. J. Clin. Nutr. 41:1289-1298.

19. Duane, W. C. 1978. Simulation of the defect of bile acid metabolism associated with cholesterol cholelithiasis by sorbitol ingestion in man. J. Lab. Clin. Med. 91:969-978.

20. Allain, C. A., L. S. Poon, C. S. G. Chan, W. Richmond, and P. C. Fu. 1974. Enzymatic determination of total serum cholesterol. Clin. Chem. 20:470475

21. McGowan, M. W., J. D. Artiss, D. R. Strandbergh, and B. Zak. 1983. A peroxidase-coupled method for the colorimetric determination of serum triglycerides. Clin. Chem. 29:538-542.

22. Duane, W. C., and S. W. Hutton. 1983. Lack of effect of experimental ascorbic acid deficiency on bile acid metabolism, sterol balance, and biliary lipid composition in man. J. Lipid Res. 24:1186-1195.

23. Miettinen, T. A., E. H. Ahrens, Jr., and S. M. Grundy. 1965. Quantitative isolation and gas-liquid chromatographic analysis of total dietary and fecal neutral steroids. J. Lipid Res. 6:411-424.

24. Grundy, S. M., and A. L. Metzger. 1972. A physiological method for estimation of hepatic secretion of biliary lipids in man. Gastroenterology. 62:1200-1217.

25. Snedecor, G. W., and W. G. Cochran. 1989. Statistical Methods, 8th ed. Iowa State University Press, Ames, IA. 217-236.

26. Keys, A. 1984. Serum cholesterol response to dietary cholesterol. Am. J. Clin. Nutr. 40:351-359.
27. Hegsted, D. M. 1986. Serum-cholesterol response to dietary cholesterol: a re-evaluation. Am. J. Clin. Nutr. 44:299-305.

28. Cobb, M. M., H. S. Teitelbaum, and J. L. Breslow, 1991. Lovastatin efficacy in reducing low-density lipoprotein cholesterol levels on high- vs. low-fat diets. J. Am. Med. Assoc. 265:997-1001.

29. DenBesten, L., W. E. Connor, and S. Bell. 1973. The effect of dietary cholesterol on the composition of human bile. Surgery (St. Louis). 73:266-273.

30. Everson, G. T., C. McKinley, and F. Kern, Jr. 1991. Mechanism of gallstone formation in women: effects of exogenous estrogen (premarin) and dietary cholesterol on hepatic lipid metabolism. J. Clin. Invest. 87:237-246.

31. Ishida, F., K. Watanabe, A. Sato, K. Taguchi, K. Kakubari, K. Kitani, and T. Kamei. 1990. Comparative effects of simvastatin (MK-733) and pravastatin (CS-514) on hypercholesterolemia induced by cholesterol feeding in rabbits. Biochim. Biophys. Acta. 1042:365-373.

32. Ishida, F., A. Sato, Y. lizuka, Y. Sawasaki, A. Aizawa, and T. Kamei. 1988. Effects of MK-733, an inhibitor of 3-hydroxy-3-methylglutaryl-coenzyme A reductase, on absorption and excretion of $\left[{ }^{3} \mathrm{H}\right]$ cholesterol in rabbits. Biochim. Biophys. Acta. 963:35-41.

33. Bates, M. C., S. G. Warren, S. Grubb, and S. Chillag. 1990. Effectiveness of low-dose lovastatin in lowering serum cholesterol. Arch. Intern. Med. 150:1947-1950.

34. Grundy, S. M., and H. Y. I. Mok. 1977. Determination of cholesterol absorption in man by intestinal perfusion. J. Lipid Res. 18:263-271.

35. Gylling, H., and T. A. Miettinen. 1992. Cholesterol absorption and synthesis related to low density lipoprotein metabolism during varying cholesterol intake in men with different apoE phenotypes. J. Lipid Res. 33:1361-1371.

36. Goodman, D. S., F. R. Smith, A. H. Seplowitz, R. Ramakrishnan, and R. B. Dell. 1980. Prediction of the parameters of whole body cholesterol metabolism in humans. J. Lipid Res. 21:699-713.

37. Kern, F., Jr. 1991. Normal plasma cholesterol in an 88-year-old man who eats 25 eggs a day. N. Engl. J. Med. 324:896-899.

38. Grundy, S. M., and G. L. Vega. 1985. Influence of mevinolin on metabolism of low density lipoproteins in primary moderate hypercholesterolemia. $J$. Lipid Res. 26:1464-1475.

39. Mistry, P., N. E. Miller, M. Laker, W. R. Hazzard, and B. Lewis. 1981. Individual variation in the effects of dietary cholesterol on plasma lipoproteins and cellular cholesterol homeostasis in man. Studies of low density lipoprotein receptor activity and 3-hydroxy-3-methylglutaryl coenzyme. A reductase activity in blood mononuclear cells. J. Clin. Invest. 67:493-502.

40. Applebaum-Bowden, D., S. M. Haffner, E. Hartsook, K. H. Luk, J. J. Albers, and W. R. Hazzard. 1984. Down-regulation of the low-density lipoprotein receptor by dietary cholesterol. Am. J. Clin. Nutr. 39:360-367.

41. Packard, C. J., L. McKinney, K. Carr, and J. Shepherd. 1983. Cholesterol feeding increases low density lipoprotein synthesis. J. Clin. Invest. 72:45-51. 